\title{
A Note on Extremality of the First Degree-Based Entropy
}

\author{
Yuhong Yang \\ College of Mathematics and Systems Science, Xinjiang University, \\ Urumqi, Xinjiang 830046, P. R. China \\ yuhong_yangsu@163.com
}

(Received April 25, 2021)

\begin{abstract}
Let $G$ be a connected graph of order $n$ with degree sequence $D(G)=\left[d_{1}, d_{2}, \ldots\right.$, $\left.d_{n}\right]$. The first degree-based entropy of $G$ is defined as

$$
I_{1}(G)=\ln \left(\sum_{i=1}^{n} d_{i}\right)-\frac{1}{\sum_{i=1}^{n} d_{i}} \sum_{i=1}^{n}\left(d_{i} \ln d_{i}\right) .
$$

In this paper, we characterize the corresponding extremal graphs which attain the maximum value of $I_{1}(G)$ among all $k$-cyclic graphs of order $n$, where $k \geq 1$.
\end{abstract}

\section{Introduction}

The information content of graphs and networks have been studied in the last fifties based on the profound and initial works due to Shannon $[11,12]$. The concept of graph entropy has been introduced to measure the structural complexity of graphs and networks $[10,13]$, which is also an information-theoretic quantity that has been introduced by Mowshowitz [9]. Moreover, many graph invariants, such as the number of vertices or edges, the vertex degree sequences, have been used for developing entropy-based measures to characterize the structure of complex network [4-7]. In this paper, we use degree powers to present graph entropies, which has been proven useful in information theory, social network, network reliability and mathematical chemistry $[1,2]$. 
Let $G=(V(G), E(G))$ be a simple connected graph with $n$ vertices and $m$ edges, where $V(G)=\{1,2, \ldots, n\}$ is called the vertex set, and the edge set $E(G)$ is composed of two-element subset $i j$ of $V(G)$ named edges, i.e., $i \sim j$ if $i j \in E(G)$. Let $N_{G}(i)=\{j \in$ $V(G) \mid j \sim i$ \} be the neighborhood of $i$ and $d_{i}=\left|N_{G}(i)\right|$ be the degree of $i$. Denote by $D(G)=\left[d_{1}, d_{2}, \ldots, d_{n}\right]$ the degree sequence of $G$. The cyclomatic number of $G$, written as $k$, is the minimum number of edges we need to remove from the graph such that the resulting graph admits no more cycles. It is well-known that $k=m-n+1$ and in this situation, $G$ is said to be a $k$-cyclic graph.

Recently, Cao, Dehmer and Shi in [3] introduced the following special degree-based graph entropy by extending the Shannon's entropy:

$$
I_{s}(G)=\ln \left(\sum_{i=1}^{n} d_{i}^{s}\right)-\frac{1}{\sum_{i=1}^{n} d_{i}^{s}} \sum_{i=1}^{n}\left(d_{i}^{s} \ln d_{i}^{s}\right) .
$$

The authors mostly analyzed the special case that $G$ is a tree, unicyclic graph, bicyclic graph for $s=1$. Therefore in this note, we consider $k$-cyclic graphs with $k \geq 1$, which generalizes the main result of Cao et al. [3].

Suppose $s=1$, observe that

$$
\sum_{i=1}^{n} d_{i}^{s}=\sum_{i=1}^{n} d_{i}=2 m
$$

From Equality (1), we infer

$$
I_{1}(G)=\ln \left(\sum_{i=1}^{n} d_{i}\right)-\frac{1}{\sum_{i=1}^{n} d_{i}} \sum_{i=1}^{n}\left(d_{i} \ln d_{i}\right)=\ln (2 m)-\frac{1}{2 m} \sum_{i=1}^{n}\left(d_{i} \ln d_{i}\right),
$$

which is called the first degree-based entropy of a connected graph by Ghalavand et al. in [8]. Therefore, if we consider the extremal values of $I_{1}(G)$ of a class of graphs with given number of edges, it suffices to determine the extremal values of $\sum_{i=1}^{n}\left(d_{i} \ln d_{i}\right)$.

Now we define a function $h(G)=\sum_{i=1}^{n}\left(d_{i} \ln d_{i}\right)$. In what follows, we consider the extremal values of $h(G)$ for $k$-cyclic graphs, from which we can easily obtain the extremal values of the graph entropy.

Let $G$ be a connected graph with $D(G)=\left[d_{1}, d_{2}, \ldots, d_{n}\right]$ such that $d_{i} \geq d_{j}+2$ for some pair of $i, j \in V(G)$, then there must exist a vertex $v \in V(G)$ such that $i \sim v$ and $j \neq v$. Let $G^{\prime}$ be the graph obtained from $G$ by removing the edge $i v$ and adding the edge $j v$. It is clear that $G^{\prime}$ has the sequence $\left[d_{1}, d_{2}, \ldots, d_{i}-1, \ldots, d_{j}+1, \ldots, d_{n}\right]$, i.e., by replacing the pair $\left(d_{i}, d_{j}\right)$ by the pair $\left(d_{i}-1, d_{j}+1\right)$. Obviously, the sum of all degree powers of $G$ 
equals the sum of all degree powers of $G^{\prime}$. The next Lemma gives a relationship between the $h(G)$ and $h\left(G^{\prime}\right)$.

Lemma 1.1 (Lemma 1, [3]). For graphs $G$ and $G^{\prime}$ shown above, we have $h(G)>h\left(G^{\prime}\right)$.

Proof. Observe that $d_{i}>d_{i}-1 \geq d_{j}+1>d_{j}$ since $d_{i} \geq d_{j}+2$. We obtain

$$
\begin{aligned}
h(G)-h\left(G^{\prime}\right) & =d_{i} \ln d_{i}+d_{j} \ln d_{j}-\left(d_{i}-1\right) \ln \left(d_{i}-1\right)-\left(d_{j}+1\right) \ln \left(d_{j}+1\right) \\
& =\left(d_{i} \ln d_{i}-\left(d_{i}-1\right) \ln \left(d_{i}-1\right)\right)-\left(\left(d_{j}+1\right) \ln \left(d_{j}+1\right)-d_{j} \ln d_{j}\right) \\
& =\left(\ln \xi_{1}+1\right)-\left(\ln \xi_{2}+1\right)>0,
\end{aligned}
$$

where $\xi_{1} \in\left(d_{i}-1, d_{i}\right)$ and $\xi_{2} \in\left(d_{j}, d_{j}+1\right)$.

\section{Extremality of the first degree-based entropy}

If $G$ is a connected $k$-cyclic graph of order $n$ having $a_{i}$ vertices of degree $d_{i}(i=1,2, \ldots, t)$, where $d_{1}>d_{2}>\cdots>d_{t}$ and $\sum_{i=1}^{t} a_{i}=n$, we write the degree sequence of $G$ as $D(G)=\left[d_{1}^{a_{1}}, d_{2}^{a_{2}}, \ldots, d_{t}^{a_{t}}\right]$.

- If $2(k-1)$ is divisible by $n$, written as $n \mid 2(k-1)$, we define a connected $r$-regular $k$-cyclic graph $G_{1}$ on $n$ vertices such that

$$
r=2+\frac{2(k-1)}{n}
$$

- If $2(k-1)$ is not divisible by $n$, written as $n \nmid 2(k-1)$, we define a connected $k$-cyclic graph $G_{2}$ of order $n$ with degree sequence $D\left(G_{2}\right)=\left[(d+1)^{a_{1}}, d^{a_{2}}\right]$, where

$$
\left\{\begin{array}{l}
d=\left\lceil 1+\frac{2 k-2}{n}\right\rceil, \\
a_{1}=(2-d) n+2 k-2, \\
a_{2}=(d-1) n-2 k+2 .
\end{array}\right.
$$

Now we aim to study the minimum value of $h(G)$ among all connected $k$-cyclic graphs.

Lemma 2.1. Let $G$ be a connected $k$-cyclic graph of order $n$, where $k \geq 1$ and $G_{1}, G_{2}$ be the graphs defined above. Then one of the following two assertions occurs:

(i) if $n \mid 2(k-1)$, it holds that $h(G) \geq h\left(G_{1}\right)$, the equality holds if and only if $G \cong G_{1}$,

(ii) if $n \nmid 2(k-1)$, it holds that $h(G) \geq h\left(G_{2}\right)$, the equality holds if and only if $G \cong G_{2}$. 
Proof. Suppose that $G_{\text {min }}$ attains the minimum value of $h(G)$ among all connected $k$ cyclic graphs of order $n$ with $m=k+n-1$ edges, and $G_{\min }$ has the degree sequence $D\left(G_{\min }\right)=\left[d_{1}, d_{2}, \ldots, d_{n}\right]$ satisfying $d_{1} \geq d_{2} \geq \cdots \geq d_{n}$. We claim that for each pair of $\left(d_{i}, d_{j}\right)$, it occurs that

$$
d_{i}=d_{j} \text { or } \quad d_{i}=d_{j}+1
$$

Otherwise if there exists a pair $\left(d_{i}, d_{j}\right)$ such that $d_{i} \geq d_{j}+2$, then there must exist a vertex $v$ such that $i \sim v$ and $j \nsim v$. We construct a $k$-cyclic graph $G^{\prime}$ obtained from $G_{\min }$ by removing the edge $i v$ and adding the edge $j v$, i.e., by replacing the pair $\left(d_{i}, d_{j}\right)$ by the pair $\left(d_{i}-1, d_{j}+1\right)$. Then by Lemma 1.1 , we obtain that $h\left(G_{\min }\right)>h\left(G^{\prime}\right)$, which contradict the minimality of $G_{\min }$. In particular, it holds that

$$
d_{1}=d_{n} \quad \text { or } \quad d_{1}=d_{n}+1 .
$$

Now we partition the discussion into the following two parts.

Case 1. $n \mid 2(k-1)$.

Let $\frac{2(k-1)}{n}=p$, where $p$ is a positive integer. We will show that $d_{1}=d_{n}$, i.e., $G_{\min }$ is a regular graph. Otherwise, by Eq. (5), it follows that $d_{1}=d_{n}+1$. So we can assume that $D\left(G_{\min }\right)=\left[\left(d_{n}+1\right)^{a_{1}}, d_{n}^{a_{2}}\right]$ with $a_{1}>0, a_{2}>0$. Obviously,

$$
\left\{\begin{array}{l}
a_{1}+a_{2}=n, \\
a_{1} \cdot\left(d_{n}+1\right)+a_{2} \cdot d_{n}=2 m=2(n+k-1),
\end{array}\right.
$$

which gives that

$$
\left\{\begin{array}{l}
a_{1}=\left(2-d_{n}\right) n+2(k-1), \\
a_{2}=\left(d_{n}-1\right) n-2 k+2 .
\end{array}\right.
$$

Since $a_{1}=\left(2-d_{n}\right) n+2(k-1)>0, a_{2}=\left(d_{n}-1\right) n-2 k+2>0$, we obtain that

$$
1+\frac{2(k-1)}{n}<d_{n}<2+\frac{2(k-1)}{n},
$$

which is equivalent to

$$
1+p<d_{n}<2+p
$$

Note that $p, d_{n}$ are all positive integers, which is impossible due to Eq. (7). Therefore $d_{1}=d_{n}$ and $G_{m i n}$ is a regular graph. Since $n d_{n}=2 m=2(k+n-1)$, then $d_{n}=2+\frac{2(k-1)}{n}$. Hence $G_{\text {min }} \cong G_{1}$. 
Case 2. $n \nmid 2(k-1)$.

Let $\frac{2(k-1)}{n}=p$, where $p$ is not an integer. We claim that $d_{1}=d_{n}+1$. Otherwise by Eq. (5), it follows that $d_{1}=d_{n}$, then $n d_{n}=2 m=2(k+n-1)$. Hence $d_{n}=2+\frac{2(k-1)}{n}=2+p$. Since $p$ is not an integer, then $d_{n}$ is also not an integer, a contradiction. Hence $d_{1}=d_{n}+1$ and we assume that $D\left(G_{\text {min }}\right)=\left[\left(d_{n}+1\right)^{a_{1}}, d_{n}^{a_{2}}\right]$ with $a_{1}>0, a_{2}>0$. Similarly, according to Eq. (6) in Case 1, we obtain that

$$
1+\frac{2(k-1)}{n}<d_{n}<2+\frac{2(k-1)}{n} .
$$

Therefore $d_{n}=\left\lceil 1+\frac{2(k-1)}{n}\right\rceil=\left\lfloor 2+\frac{2(k-1)}{n}\right\rfloor$ and

$$
\left\{\begin{array}{l}
a_{1}=\left(2-\left\lceil 1+\frac{2(k-1)}{n}\right\rceil\right) n+2(k-1) \\
a_{2}=\left(\left\lceil 1+\frac{2(k-1)}{n}\right\rceil-1\right) n-2 k+2
\end{array}\right.
$$

which gives that

$$
D\left(G_{\text {min }}\right)=\left[\left(\left\lceil 1+\frac{2(k-1)}{n}\right\rceil+1\right)^{\left(2-\left\lceil 1+\frac{2(k-1)}{n}\right\rceil\right) n+2(k-1)},\left\lceil 1+\frac{2(k-1)}{n}\right\rceil^{\left(\left\lceil 1+\frac{2(k-1)}{n}\right\rceil-1\right) n-2 k+2}\right] .
$$

Hence $G_{\min } \cong G_{2}$.

Combining Lemma 2.1 with Eq. (2), we finally get the following result towards the extremal properties of the graph entropy.

Theorem 2.1. Let $G$ be a connected $k$-cyclic graph $(k \geq 1)$ with $n$ vertices. Then one of the following conditions holds:

(i) if $n \mid 2(k-1), I_{1}(G) \leq I_{1}\left(G_{1}\right)$, the equality holds if and only if $G \cong G_{1}$,

(ii) if $n \nmid 2(k-1), I_{1}(G) \leq I_{1}\left(G_{2}\right)$, the equality holds if and only if $G \cong G_{2}$.

Remark 1. Let $G$ be a connected $k$-cyclic graph of order $n$ with degree sequence $D(G)=$ $\left[d_{1}, d_{2}, \ldots, d_{n}\right]$.

- If $n \mid 2(k-1)$ and $G$ is not isomorphic to $G_{1}$, together with Case 1 in the proof of Lemma 2.1, we observe that $d_{1} \neq d_{n}+1$. Then we deduce that there exists a pair $\left(d_{i}, d_{j}\right)$ such that $d_{i} \geq d_{j}+2$. In this situation, there also exists a vertex $v \in V(G)$ satisfying $i \sim v$ and $j \nsim v$. Let $G^{*}$ be the graph obtained from $G$ by removing the edge iv and adding the edge $j v$. Continue this process until there is no pair $\left(d_{i}, d_{j}\right)$ such that $d_{i} \geq d_{j}+2$. Thus we obtain a $k$-cyclic graph sequence $G, G^{*}, G_{1}^{*}, \ldots, G_{s}^{*}$ such that $G_{s}^{*} \cong G_{1}$. 
- If $n \nmid 2(k-1)$ and $G$ is not isomorphic to $G_{2}$, after the same operation as above, we can obtain a graph $G_{s}^{* *}$ such that for each pair $\left(d_{i}, d_{j}\right)$, it occurs that $d_{i}=d_{j}$ or $d_{i}=d_{j}+1$. Combining with Case 2 in the proof of Lemma 2.1, we have $d_{1}=d_{n}+1$ and $G_{s}^{* *} \cong G_{2}$.

Now we consider two particular cases in which $k \in\{1,2\}$. We conclude this paper by the following two Corollaries, which can be deduced from the result of [3].

Corollary 2.1 (Theorem 2, [3]). Let $G$ be a unicyclic graph(1-cyclic graph) of order $n$, then $I_{1}(G) \leq I_{1}\left(C_{n}\right)$, where $C_{n}$ is a cycle of order $n$. The equality holds if and only if $G \cong C_{n}$.

Proof. By Theorem 2.1, if $k=1$, then for each unicyclic graph of order $n$, it holds that $n \mid 2(k-1)=0$. According to Eq. (3), the corresponding extremal graph $G_{1}$ is a 2 -regular unicyclic graph, i.e., $G_{1}$ is a cycle.

Corollary 2.2 (Theorem 3, [3]). Let $G$ be a bicyclic graph(2-cyclic graph) of order $n$, then $I_{1}(G) \leq I_{1}\left(G_{2}\right)$, where $G_{2}$ is a connected bicyclic graph with degree sequence $D\left(G_{2}\right)=$ $\left[3^{2}, 2^{n-2}\right]$. The equality holds if and only if $G \cong G_{2}$.

Proof. By Theorem 2.1, if $k=2$, then for each bicyclic graph of order $n(n>2)$, it follows that $n \nmid 2(k-1)=2$. From Eq. (4), we have $d=2, a_{1}=2, a_{2}=n-2$. Then the corresponding extremal graph $G_{2}$ is an irregular graph with degree sequence $D\left(G_{2}\right)=\left[3^{2}, 2^{n-2}\right]$.

\section{References}

[1] B. Bollobás, V. Nikiforov, Degree powers in graphs with forbidden subgraphs, El. J. Comb. 11 (2004) \#R42.

[2] B. Bollobás, V. Nikiforov, Degree powers in graphs: The Erdős-Stone theorem, Comb. Prob. Comput. 21 (2012) 89-105.

[3] S. Cao, M. Dehmer, Y. Shi, Extremality of degree-based graph entropies, Inf. Sci. 278 (2014) 22-33.

[4] Z. Chen, M. Dehmer, Y. Shi, A note on distance-based graph entropies, Entropy 16 (2014) 5416-5427. 
[5] M. Dehmer, Information processing in complex networks: Graph entropy and information functionals, Appl. Math. Comput. 201 (2008) 82-94.

[6] M. Dehmer, A. Mowshowitz, A history of graph entropy measures, Inf. Sci. 181 (2011) 57-78.

[7] M. Eliasi, On extremal properties of general graph entropies, MATCH Commun. Math. Comput. Chem. 79 (2018) 645-657.

[8] A. Ghalavand, M. Eliasi, A. Ashrafi, First degree-based entropy of graphs, J. Appl. Math. Comput. 59 (2019) 37-46.

[9] A. Mowshowitz, Entropy and the complexity of graphs. I. An index of the relative complexity of a graph, Bull. Math. Biophys. 30 (1968) 175-204.

[10] N. Rashevsky, Life, information theory, and topology, Bull. Math. Biophys. 17 (1955) $229-235$.

[11] C. E. Shannon, W. Weaver, The Mathematical Theory of Communication, Univ. Illinois Press, Urbana, 1949.

[12] C. E. Shannon, A mathematical theory of communication, Bell Syst. Tech. J. 27 (1948) 379-423.

[13] E. Trucco, A note on the information content of graphs, Bull. Math. Biophys. 18 (1965) 129-135. 